\title{
FPGA Based Real Time Medical Image Processing
}

\author{
Akshay S. Janmane, Apeksha S. Patil and Madhuri V. Huilgol
}

\begin{abstract}
The digitisation of medical images tend to suffer from poor image quality, lack of proper contrast and most importantly presence of shading and artefacts In order to overcome the poor image quality of the image, enhancement is the key method which is followed. Enhancement improves the effectiveness or attractiveness of the image. Enhancement involves conversion of image from gray to adoptive histogram and then $2 D-D W T$ is applied on it. This decomposes the image into 4 sub-bands and the low frequency sub band is smoothened using geometric mean filter. Then IDWT is applied using new low frequency and remaining sub bands. The experimental results vividly displays the proposed algorithm is efficient to enough to remove the noise resulting good enhancement and then achieved increased value of PSNR and reducing value of MSE. In order to effectively present both gray values and spatial information of image pixels, a fusion method for image segmentation by jointly utilizing direct fusion method and edge detection method is being proposed. The technique involved here is division of image into small sub blocks each sub block consisting of a vector and those vectors are further classified as edge and non-edge patterns by using edge detection algorithm. Boundary detection is further applied to extract the finest boundary curves in the edge pattern vectors and finally a fusion method is employed to synthesise the results of Pixel Based Fusion Method and Boundary Detection to accomplish the segmentation.
\end{abstract}

Keywords--- Geometric Mean Filter, Image Segmentation, Edge Detection, Information Fusion.

\section{INTRODUCTION}

$\mathrm{E}$ NHANCEMENT plays a very important aspect in image processing; that is improving the quality of an image is named as enhancement. Image enhancement is the process of adjusting digital images so that the results are more suitable for present or more image investigation. Image enhancement [1] refers to emphasis, sharpening of image features such as edges, borders, or difference to

Build a graphic display more useful for display and investigation. Image improvement includes grey level and contrast operation, noise reduction, edging, sharpening, filtering, interpolation and intensification, pseudo colouring, and so on. However, the image improvement is based on

Akshay S. Janmane, Student, Department of ECE, SDMCET, India. E-mail:akshayjanmane@gmail.com

Apeksha S. Patil, Student, Department of ECE, SDMCET, India. E-mail:apeksha26091995@gmail.com

Madhuri V. Huilgol, Department of ECE, SDMCET, India. E-mail:madhurihuilgol@gmail.com

DOI:10.9756/BIJRCE.8214 arithmetic mean and variance calculation. While an arithmetic mean filter smoothens neighbouring variant in an image and noise is condensed to some extent, it actually results in blurring of the image. Also, the arithmetic mean filter tends to lose image particulars such as edges and sharpness.

Image segmentation is defined as the process of dividing an image into several meaningful segments for extended analysis [2]. We find about seven segmentation methods including statistical probability of pixel characteristic based method[3], region related segmentation methods, clustering methods, graph theory based methods, level set methods [4,5], hybrid methods [6], and fuzzy theory based methods. Most of the methods concentrate on the gray value information than the geometrical structure information [7-9].Human vision perception of image content is actually based on learning local region features and their internal structures instead of pixel features and heir gray value information. Therefore it becomes very important to design a segmentation method which can effectively make use of both spatial structural information of local regions and gray value information of pixels.

This method mainly involves sub blocks which are mainly divided from an image processing objects and this uses both the spatial structure information and gray value information to form the feature vectors of sub-blocks. Initially the feature vectors are mainly classified into two patterns which are named as Edge pattern and Non-edge pattern respectively. This is achieved by edge detection system. Further the Boundary detection algorithm is mainly developed to extract the refined boundary curves of the edge pattern sub-block vectors and Pixel based fusion method (PBFM) is mainly presented for segmenting the non-edge pattern sub-block vectors. During the concluding part segmentation result is obtained by information fusion which involves PBFM and BD results.

\section{IMAGE ENHANCEMENT}

Initially Adoptive Histogram is applied to grey medical image. After this process Discrete wavelet transform-DWT is applied to this image which converts it from spatial field to frequency field. The image can then be separated into four different bands i.e. LL, LH, HL, and HH as shown in figure 1. LL band is chosen for further analysis because of lower bandwidth.

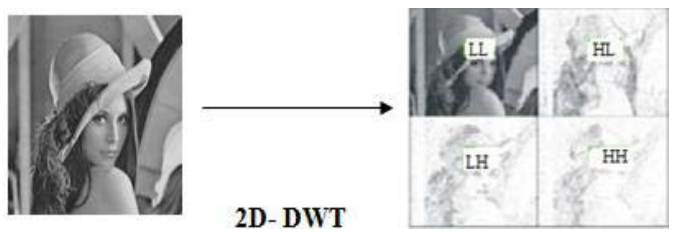

Figure 1: Frequency Distribution of DWT 
The Geometric mean is defined as a group of average of a setoff number that is distinct from the arithmetic average. This is computed by multiplying all the numbers, and taking the nth root of the total. The geometric mean between two numbers and it is implemented using (1).

$$
\text { xgeom }=\sqrt[n]{\prod_{i=1}^{n} x_{i}}=n \sqrt[n]{x_{1} \cdot x_{2} \ldots . . x_{n}}=G M=\left(\left(x_{1}\right)\left(x_{2}\right)\left(x_{3}\right) . .\right.
$$

Where GM is Geometric Mean and $x=$ indivıuua sore and $n=$ sample size (Number of scores).Then the IDWT is applies and we get the enhanced image.

\section{IMAGE SEGMENTATION}

The method of edge detection is achieved using 2 steps: firstly the original image has to be pre-processed and secondly an edge pattern discriminator is designed to divide edge pattern and non-edge pattern vector. This is represented below:

\section{A. Pre-Processing}

The image to be segmented is pre-processed, and the edge information of the image is enhanced, while non-edge and noise information is restricted. The wavelet functions are defined as:

$$
\phi^{1}(x, y)=\frac{d}{d x} \theta(x, y), \phi^{2}(x, y)=\frac{d}{d y} \theta(x, y)
$$

$\theta(\mathrm{x}, \mathrm{y})$ is a two-dimensional smooth function:

$$
\iint_{R R} \theta(x, y) d x d y=1, \lim \theta(x, y)=0
$$

Set the scale factor $\mathrm{a}=2^{\mathrm{j}}$, the two-dimensional wavelet function base could be denoted as:

$$
\phi_{2 j}^{1}(x, y)=\frac{1}{2^{2 j}} \phi^{1}\left[\frac{x}{2 j}, \frac{y}{2 j}\right], \phi_{2 j}^{2}(x, y)=\frac{1}{2^{2 j}} \phi^{2}\left[\frac{x}{2^{j}}, \frac{y}{2^{j}}\right]
$$

The original image is represented by $\mathrm{t}(\mathrm{x}, \mathrm{y})$, and the horizontal and vertical edge information of $\mathrm{f}(\mathrm{x}, \mathrm{y})$ at scale $\mathrm{a}=$ $2^{\mathrm{j}}$ could be calculated following Eq. (5).

$$
\begin{aligned}
& W_{2 j}^{1} f(x, y)=f(x, y) \otimes \phi_{2 j}^{1}(x, y), \\
& W_{2 j}^{2} f(x, y)=f(x, y) \otimes \phi_{2 j}^{2}(x, y),
\end{aligned}
$$

Therefore, the modulus and angles of image gradient vectors could be calculated by Eq. (6) [10-11]

$$
\begin{gathered}
M_{2 j} f(x, y)=\sqrt{\left|W_{2 j}^{1} f(x, y)\right|^{2}+\left|W_{2 j}^{2} f(x, y)\right|^{2}} \\
A_{2 j} f(x, y)=\arctan \left[\frac{W_{2 j}^{2} f(x, y)}{W_{2 j}^{1} f(x, y)}\right]
\end{gathered}
$$

Based on the above calculation, the proposed preprocessing method is composed of the following three steps:
1. Divide the scale of gradient vector angle into four ranges as shown in Fig. 2a: horizontal region $\left(0^{\circ}\right.$ and $180^{\circ}$ direction), right diagonal region $\left(45^{\circ}\right.$ and $225^{\circ}$ direction), vertical region ( $90^{\circ}$ and $270^{\circ}$ direction), and left diagonal region ( $135^{\circ}$ and $315^{\circ}$ direction).

2. Calculate the modulus value $\mathrm{M}_{2 \mathrm{j}} \mathrm{f}(\mathrm{x}, \mathrm{y})$ and angle value $A_{2 j} f(x, y)$ of each pixel.

3. As demonstrated in Fig. 2b, pixel 5 is standing for the current processed pixel, and others are standing for its 8-neighborhood pixels. Examine the angle direction $A_{2 j} f(x, y)$ of pixel 5, and compare the modulus values of the 3 pixels which are on that direction, if the modulus values of pixel 5 is the greatest, add the 2 modulus values which are on the vertical direction of $A_{2 j} f(x, y)$ to the modulus values of pixel 5 , this means pixel 5 is a potential edge point. Otherwise keep the modulus value unchanged which indicates the pixel 5 is non-edge point. In this way, due to the consideration of local structure of pixel's neighbourhood, the modulus values of edge pixels are enhanced, while the non-edge pixels and noise pixels are not.

\section{B. Edge Pattern Detection}

The main steps involved in the proposed edge pattern detection algorithm for discriminating the edge pattern vectors and non-edge pattern vectors based on the pre-processed image are as follows:

1. Transform the pre-processed image into the formation of sub-blocks set (size 3X3).

2. Then calculate the mean modulus value of the preprocessed image, which will then be considered as the threshold $\mathrm{T}$ for edge pattern detection.

3. Then calculate the mean modulus value of the three pixels which are on the vertical direction of the angle direction of pixel 5 for each sub-block.

4. Perform the comparison for the mean value of each sub-block with the threshold $\mathrm{T}$, and the sub-block would be identified as edge pattern if its mean value was greater than threshold $T$. The non-edge pattern sub-blocks could also be detected correspondingly and that would have a reverse condition.

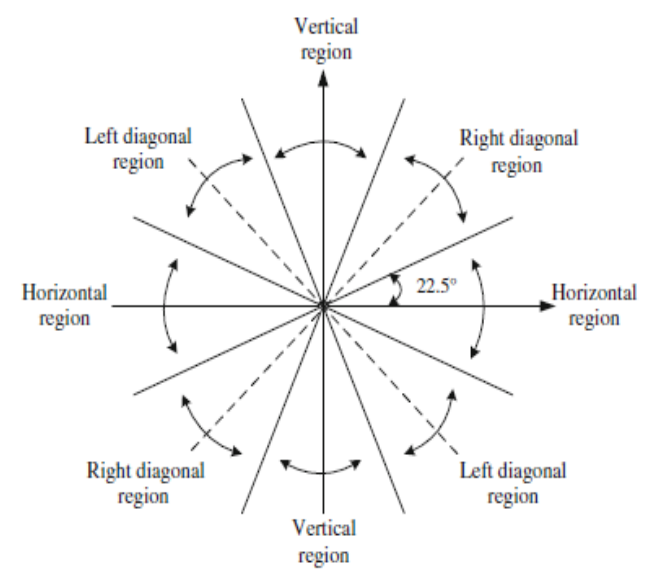

Figure 2: Diagram of Angle Directions Division 
4 Angle Regions Division

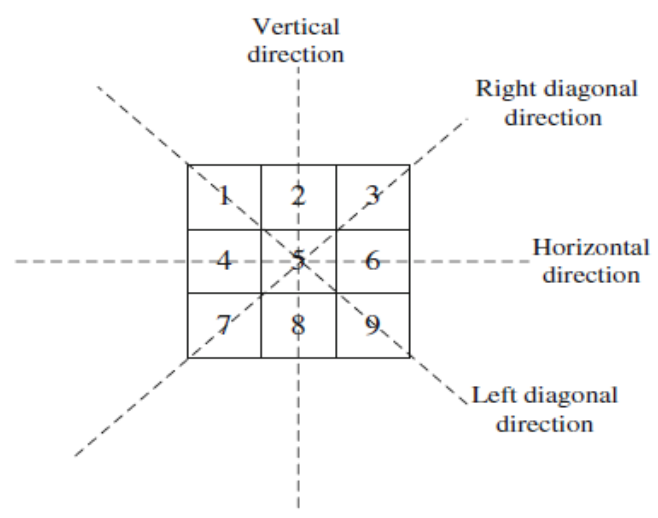

Figure 3:The 8-Neighborhood Pixels According to Angle Division

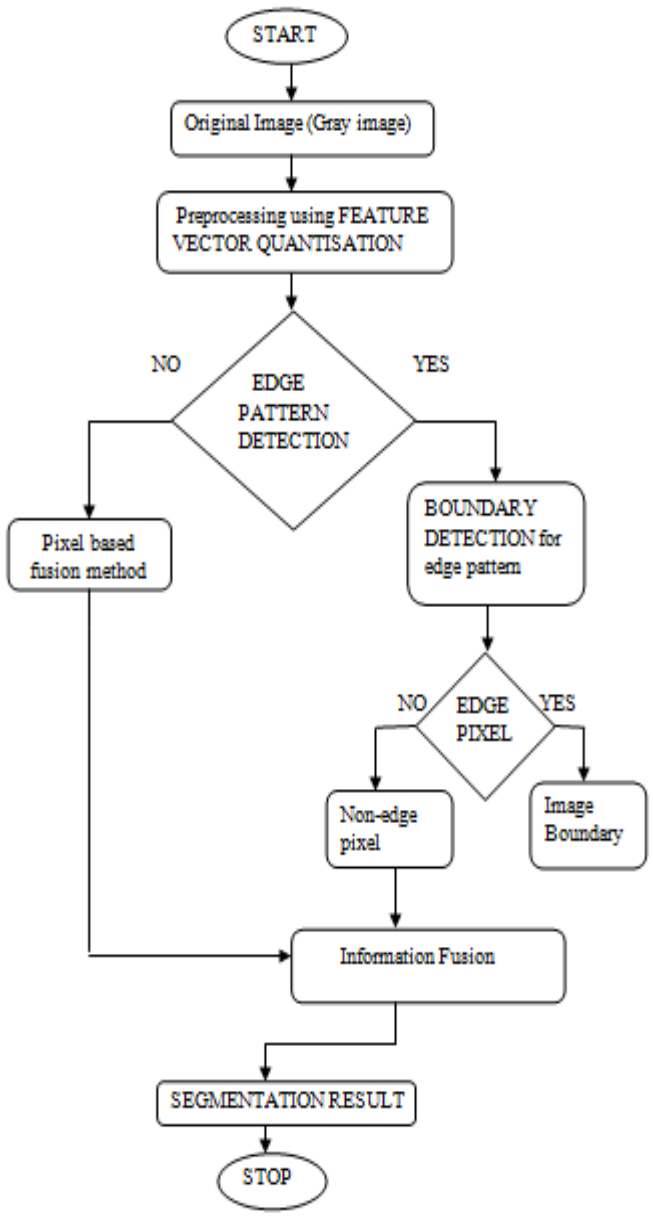

Figure 4: Flow Chart of Proposed Method

\section{Pixel BAsed IMAge Fusion Method}

Image fusion process mainly involves fusing of 2 or more images and then synthesizes them into one that contains all the significant or clear information from every input image. The process of image fusion can be mainly classified into 1.Region based 2.Pixel based and 3.Decision based and here we are proposing the pixel based fusion method.
The detail information that is extracted from one image using wavelet transforms can be injected into another image using one of a number of methods, for example substitution, addition, or a selection method based on either frequency or spatial domain.

\section{Image Segmentation Process}

After the sub-block vectors have been discriminated to edge pattern and non-edge pattern, then comes the segmentation process on the non-edge pattern vectors using Pixel based Fusion Method and perform Edge Detection on the edge pattern vectors. In this section, we will present the specified steps of the proposed segmentation method based on the fusion of vector quantization and edge detection.

\section{A. Edge Pattern Processing}

After processing non-edge pattern vectors through PBFM method, the edge pattern processing is done to further analyse edge-pattern sub-blocks and finally achieve the task of boundary extraction and also to provide further information for fusion purpose.

\section{B. Boundary Detection}

During the Boundary detection the refined curves of the image are properly extracted by further analysis on local pixels of edge-pattern sub-blocks.

1. Calculate the mean modulus value $\mathrm{TM}_{\mathrm{m}}$ of each edge pattern sub-block.

2. Compare TM with the modulus value of each sub-block pixel, and the edge pixel could be identified if its modulus value is larger, which would be set to 255 correspondingly. Otherwise, the pixel will be viewed as non-edge pixel, which would be set to 0 .

\section{Information Fusion}

The information fusion between the results of PBFM of non-edge pattern sub-block and BD of edge-pattern sub-block provides an accurate result of segmentation. The fusion method here is:

1. For the PBFM result of non-edge pattern sub-blocks: The mean value of each sub-block is calculated.

2. For BD result of edge pattern sub-blocks: The nonedge pixels are left after boundary extraction, examine the calculated mean values of the non-edge sub blocks (from (i)) which are neighbouring with those non-edge pixels, and locate the non-edge subblock that has the minimum value difference with the non-edge pixel.

3. The value of the non-edge pixel would be set to the Mean value of the cluster that the located non-edge sub-block is belonging to, namely the pixel is classified into that cluster.

$$
\text { VI. RESUlts }
$$

\section{Segmentation}

A small demonstration of the proposed algorithm is shown in Figure 5 in which an image that was chosen was a human brain image and that is segmented. In this segmentation algorithm the image is mainly segmented into 3 main classes that comprises of white matter, gray matter and background. 
In order to display each class clearly the pixels of the displayed class are set to a representative value which forms the code word of this class and all others are set to zero. The boundary result of wavelet modulus maxima edge detection is shown in Fig. 5b, the edge pattern detection result is shown in Fig. 5c, the boundary extraction result is shown in Fig. 5d, the brain white matter segmentation result after information fusion of PBFM result and edge detection result is shown in Fig. 5e, and the brain grey matter segmentation result is shown in Fig. 5f. Different with traditional segmentation method, in additional of segmentation result, the proposed method presents image boundary information. By comparing the Fig. $5 \mathrm{~b}$, d, we can see that the proposed method (shown in Fig. 5d) outperforms the wavelet modulus maxima edge detection method (shown in Fig. 5b). The information fusion result (shown in Fig. 5e, f) is satisfying as well.

The grey medical image is first enhanced and then the image is segmented to extract the required data. The proposed idea is to be implemented on FPGA evaluation board.

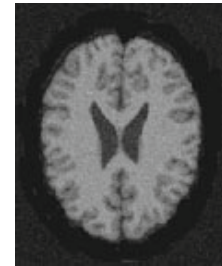

(a)

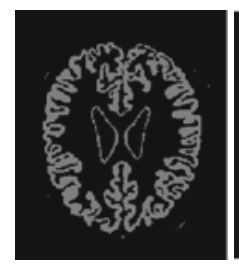

(d)

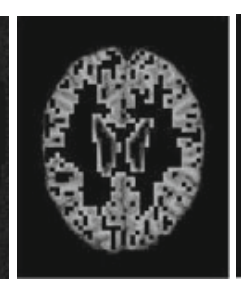

(b)

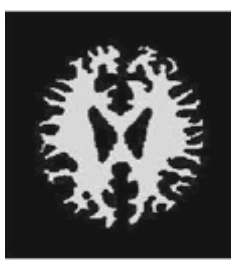

(e)

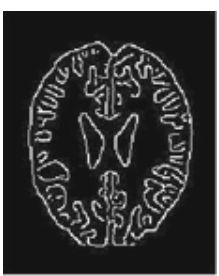

(c)

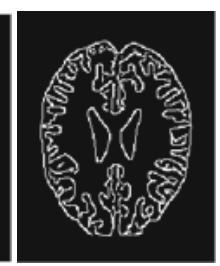

(f)
Figure 5: Expected Results. a)Original image; b) Result of Wavelet Modulus Maxima Edge Extraction; c) Result of edge Pattern Detection; d) Result of Boundary Extraction; e) Result of White Matter; f) Result of Grey Matter

\section{REFERENCES}

[1] R.C. Gonzalez and R.E. Woods, "Digital Image Processing Reading, MA: Addison-Wesley, 1992.

[2] Y.J. Zhang, “Image segmentation”, Science Press, Beijing, 2001.

[3] X.L. Gao, Z.L. Wang and J.W. Liu, “Algorithm for image segmentation using statistical models based on intensity features”, Acta Optica Sinica, Vol.31, No.1, Pp.1-6, 2011.

[4] J. Zhao, F.Q. Shao and X.D. Zhang, "Vector-valued images segmentation based on improved variational GAC model”, Control Decision, Vol.26, No.6, Pp.909-915,2011.

[5] J. Zhao, F.Q. Shao and X.D. Zhang, "A fast C-V model for vector valued image segmentation”, J Comput Aided Design Comput Gr, Vol.23, No.11, Pp.1869-1874, 2011.

[6] Y. Wu, P. Xiao and C.M. Wang, "Segmentation algorithm for SAR images based on the persistence and clustering in the contour let domain”, Acta Optica Sinica, Vol.30, No.7, Pp.1977-1983, 2010.

[7] J. Thornton and A. Srbic, "Spatial pooling for greyscale images”, Int J Mach Learn Cybern, Vol.4, No.3, Pp.207-216, 2013.

[8] J. Gamio, S. Belongie and S. Majumdar, "Normalized cuts in 3D for spinal MRI segmentation”, IEEE Trans Med Imaging, Vol.23, No.1, Pp.36-44, 2004.
[9] O. Veksler, “Image segmentation by nested cuts”, In: Proceedings of IEEE Conference on Computer Vision and Pattern Recognition. IEEE Press, Hoboken, Pp.339-344, 2000.

[10] http://www.indjst.org/index.php/indjst/article/viewFile/56192/64987

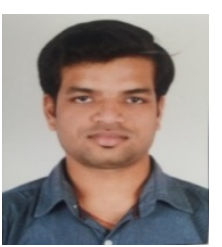

Akshay S. Janmane, born in Shimoga on $20^{\text {th }}$ of April, 1995.Persued primary Education in SDM English medium HS, Dharwad. Persued higher Secondary Education in JSS college, Dharwad. Persuing $7^{\text {th }}$ semester, B.E in Electronics and Communication Engineering at SDMCET, Dharwad.

(E-mail:akshayjanmane@gmail.com)

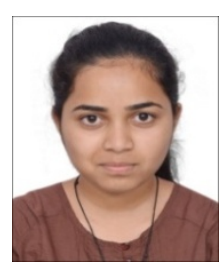

Apeksha S. Patil, born in Dharwad on $26^{\text {th }}$ of september,1995. Persued primary Education in GSES English medium HS, Chikodi, Belgaum.Persued higher Secondary Education in JSS college, Dharwad. Persuing $7^{\text {th }}$ semester B.Ein Electronics and Communication Engineering at SDMCET, Dharwad.

(E-mail:apeksha26091995@gmail.com)

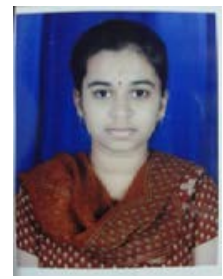

Madhuri V. Huilgol, born in Hubli on $20^{\text {th }}$ of April,1995. Persued primary Education in Presentation Girls HS, Dharwad. Persued higher Secondary Education in KCD college, Dharwad. Persuing $7^{\text {th }}$ semester B.E in Electronics and Communication Engineering at SDMCET, Dharwad.

(E-mail:madhurihuilgol@gmail.com) 